\title{
Simulation-Based Investigation of the Influence of Process Parameter Deviations on the Quality of Clinch Connections with Preformed Hole
}

\author{
Bernd Maier ${ }^{1, a^{*}}$, Markus Klingler ${ }^{1, b}$, Sabine Böhm ${ }^{2, c}$ and Birgit Awiszus ${ }^{3, d}$ \\ ${ }^{1}$ Robert Bosch $\mathrm{GmbH}$, Automotive Electronics, 72770 Reutlingen, Germany \\ ${ }^{2}$ Robert Bosch $\mathrm{GmbH}$, Automotive Electronics, 71701 Schwieberdingen, Germany \\ ${ }^{3}$ Chemnitz University of Technology, Department of Virtual Production Engineering, 09126 \\ Chemnitz, Germany \\ aBernd.Maier3@de.bosch.de, ’ Markus.Klingler@de.bosch.com, 'Sabine.Boehm3@de.bosch.com, \\ dbirgit.awiszus@mb.tu-chemnitz.de
}

Keywords: Clinching, Forming simulation, process parameter tolerances.

\begin{abstract}
In this work, the influences of deviations of material properties (used material is aluminium for both metal sheets), hole geometry (diameter, chamfer at the bottom and rounding at the top) and offset between punch and hole on the quality of a clinched connection are analysed. The analyses were done with numerical forming simulations, which were validated by experimental tests. For each process parameter, models were built up to simulate the forming process. After simulation of the forming process, it was possible to measure the resulting undercut and to identify the dependency between process parameters and width of undercut. This shows the influence of each investigated parameter on clinch quality and enables to set tolerances as high as possible but small enough to get the required undercut in the clinched connection.
\end{abstract}

\section{Introduction}

The simulation-based analysis of manufacturing process parameters is getting more and more important. With FEM, it is possible to simulate the whole manufacturing process before physical specimens are built up. This helps to save a lot of time and money during the design development. Additionally it is possible to simulate the influence of tolerances of the process parameters. This is very helpful to fix the necessary tolerance for a successful working process. The mechanical joining process, clinching, is a manufacturing process in which forming simulations can help a lot

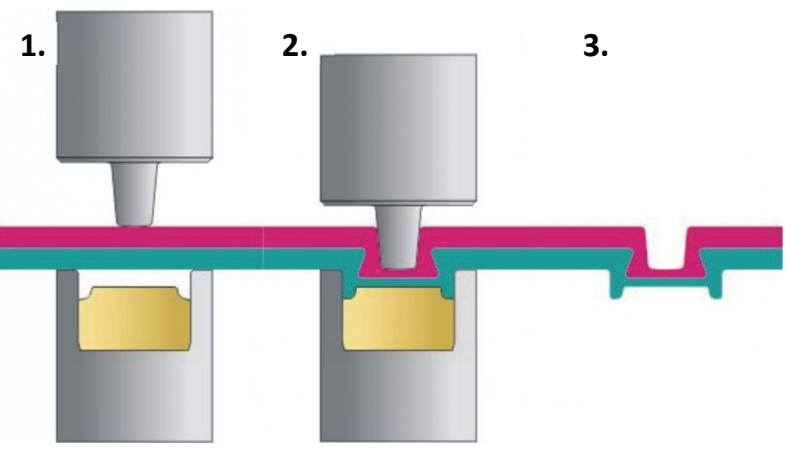

Fig. 1. Sequences of a no-cutting, single part die clinching process [3]. in process development. Clinching is a very interesting joining technique because of the advantages that there is no need of additional joining parts like screws, there is no emission of welding gases during the clinching process, there is no heat injection in the joining partners and many more. [1,2]

The joining process is illustrated in picture 1. To get an interlocked connection of the sheet metal joining partners, a punch and a die is used in a forming process. In this process, the die is placed under the bottom metal sheet. During the joining process the punch which is placed above the upper metal sheet moves downwards. This punch presses the material of the top metal sheet into the die. The geometry of the punch and die is tuned in a way that an interlocked connection between the two metal sheets is formed. To develop that punch and die geometry forming simulations can be very helpful. $[4,5,6,7]$ 

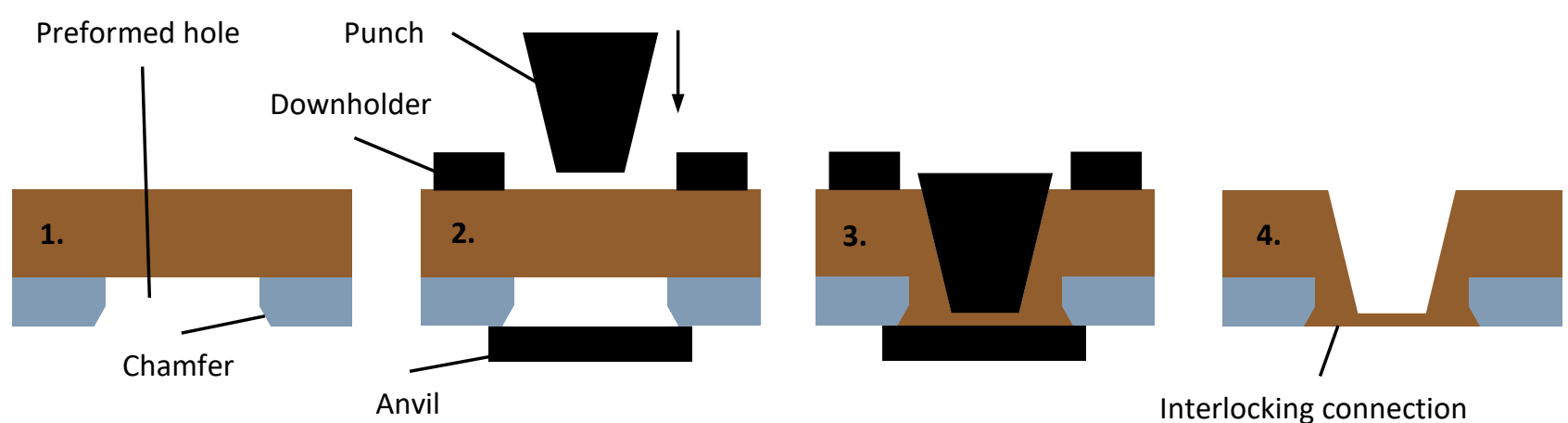

Interlocking connection

Fig. 2. Special clinching method with preformed hole on bottom side metal sheet (schematic).

Special Clinching Method. In this work, a special clinching variant with a preformed hole was examined. The used clinching method is shown in picture 2. It was not possible to use a standard clinching method with a single or a multiple part die to connect the two meatal sheet parts because there was not enough space to place a die. The available space was sufficient only to place a flat anvil. The bottom metal sheet was provided with a preformed hole including a chamfer at the lower side of the hole. The material of the upper metal sheet was formed through the hole, during the clinching process, and behind the chamfer. So it is possible to build up an interlocking connection.

Quality criterion. The used quality criterion of the clinched connection in this work is the undercut which builds up the interlocking connection. The needed undercut for a working connection was measured in tests. The value of this undercut (see Fig. 5), which is needed in the product was normalized to the value of 1 . So for this paper an undercut of 1 around the clinch has to be created to ensure that the interlock of the connection is wide enough and the connection of the two joining partners won't fail. Unbuttoning was the only detected failure in the tensile test of the clinched connection. Because of this it is the only quality criterion which was analysed and other failures of clinched connections like neck cracks were neglected.

\section{Research Method}

Process forming simulations with an FE-Software was the mainly used research method for this paper. The used Software were Ansys for the analysis of 2D-Models and LS-Dyna to build up the forming process in 3D. The 3D-Models were necessary to simulate the forming process with an offset between punch and preformed hole axes, because those problems are no longer axially symmetric. To validate the simulated results, samples were built up. This way, simulated and real clinch geometries could be compared, as well as the simulated and real force-displacement curves of the clinching process. With those two components, it is possible to ensure that the simulation model is working correctly.

Geometry setting. In this work changes in geometry of the preformed hole are analysed. Picture 3 shows the geometry of the preformed hole. The four Parameters A, B, C and D describe the geometry of the hole. The hole radius is represented by parameter A. Parameters B and C describe the shape of the chamfer at the bottom of the hole and the parameter $\mathrm{D}$ the radius at the top of the hole. For each parameter are value and tolerances are defined which are given by the manufacturability of the hole.

A second geometry influence which was researched is an offset between the axes of punch and the preformed hole. That offset is given by

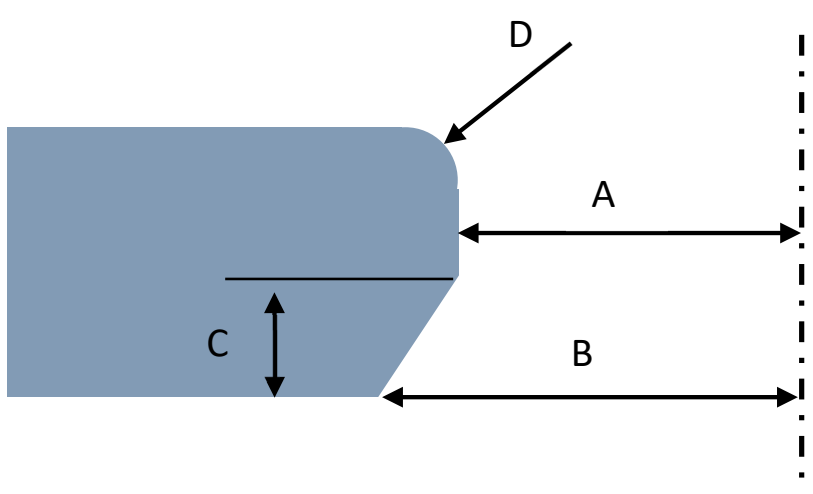

Fig. 3. Hole geometry in bottom metal sheet. 


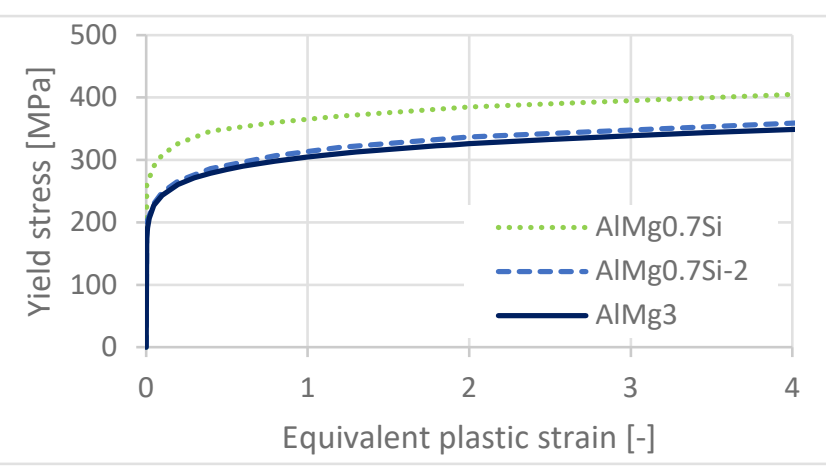

Fig. 4. Used hardening curves for simulation.

the fact, that it is not possible to insert the two sheet metal parts perfectly in the clinching machine. Because of that fact there is also a tolerance defined for the offset between punch and preformed hole.

Material and Data. The used material in the work was AlMg3 for the bottom metal sheet and $\mathrm{AlMg} 0.7 \mathrm{Si}$ for the upper metal sheet. Because of tolerance in a heat treatment process of the upper metal sheet there are also little tolerances in the material properties of that component. Important for the forming process are the changes in the hardening curves of the metal sheet. In this work two different hardening curves for the upper metal sheet were used to analyse the influence on the clinching process. The hardening curve of the bottom metal sheet was kept the same. The hardening curves are shown in figure 4 . The conventionally used hardening curve for the upper metal sheet is the curve of AlMg0.7Si from picture 3. The hardening curve AlMg0.7Si-2 was only used for the comparison of different material data.

Forming simulation. To simulate the forming process two different FE software programs were used. Ansys was used for the 2D simulations and LS-Dyna for the 3D simulations. With the implicit solver of Ansys it is possible to get the results very fast. But because of the high deformations of the mesh it is necessary to use a remeshing tool during the forming simulation. Those remeshing tools only work smoothly for 2D problems. Because of this, the explicit solver of LS-Dyna was used for the 3D forming simulations. With this software there are no problems when the mesh gets highly deformed. Unfortunately the simulation time is much longer with the explicit LS-Dyna than with the implicit Ansys solver. For both simulations models the same boundary conditions were used. The materials of the two joining partners were modelled with a multilinear plasticity model. The used hardening curves are shown in figure 4 . The forming tools were modelled with an elastic material model with an Young's modulus of $210 \mathrm{GPa}$. The coulomb law of friction was used to model friction effects between the different parts. The used friction parameters were $\mu=0.2$ between the two joining partners and $\mu=0.15$ between aluminium metal sheets and tools, except between punch and

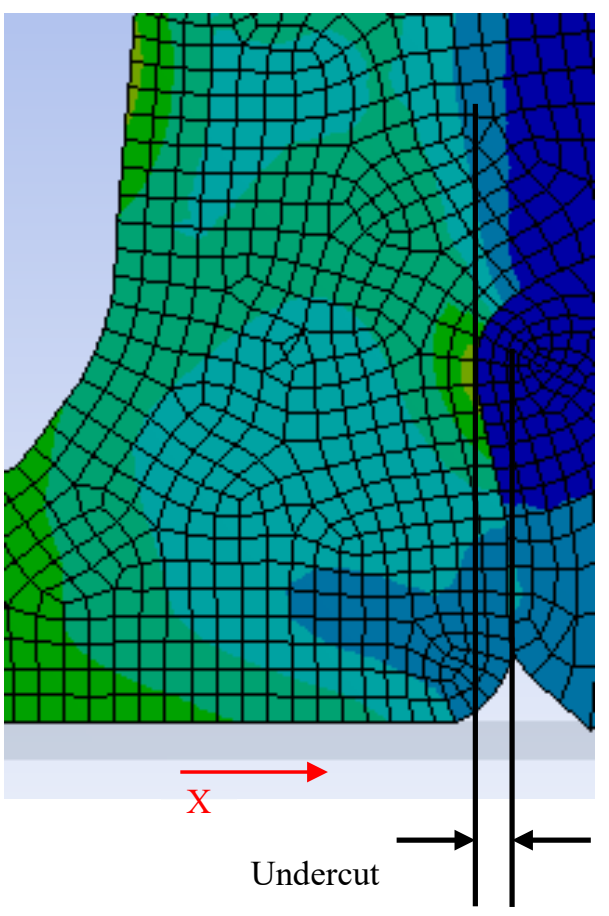

Fig. 5. Undercut measurement. aluminium metal sheet. Between those two parts a friction value of $\mu=0.1$ was set because of the fact that the punch is lubricated with oil before each clinching process.

Undercut measurement. The width of undercut was used to qualify the clinched connection. Test have shown that a minimum undercut width is required to ensure a sufficiently reliable connection. The formed undercut can be measured in cross sections for the physical clinches. The measurement in the simulation is much easier (Picture 5). The undercut was defined as the differences between the lowest value of $\mathrm{x}$-coordinate of the nodes of the bottom metal sheet and the highest value of $\mathrm{x}$ coordinate of the nodes of the upper metal sheet in a fixed area.

\section{Results and Discussion}

To ensures that the simulated results are right it is important to validate the simulation model. For this validation the simulated results are compared with physical tests. After successful validation of the numerical model, it could be used to analyse the influence of the researched process parameters on the clinch quality. 


\section{Validation of simulation}

model. To validate the numerical model of the clinch forming process two comparison have been done. Firstly, the simulated geometry after clinching was compared with a cross section of the physical clinch. Figure 6 shows the simulated geometry on the left side of the picture and the cross section of the physical clinch on the right side of the picture. The comparison shows that the simulation is able to calculate the

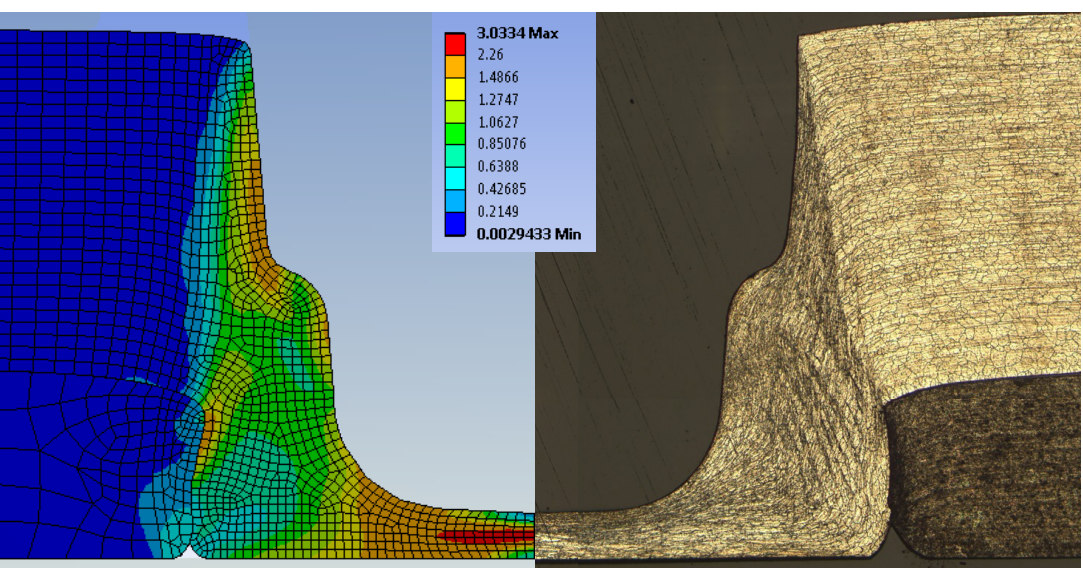

Fig. 6. Comparison of the simulated geometry (2D) and a cross section of the clinch. deformed contour after the clinching process correctly. In addition to the contour of the deformed material, the picture shows the local deformations in the clinch. In the simulated picture, plastic strain is given by the different colours. In the cross section the local deformation is visible from the deformed grains of the metal. The comparison of the two pictures shows that the used numerical model is able to simulate the correct local deformations and contour of the clinching process. The comparison has been done for both models, 2D and 3D. It is only shown for the 2D model in this paper, but the 3D model also gives the same results.

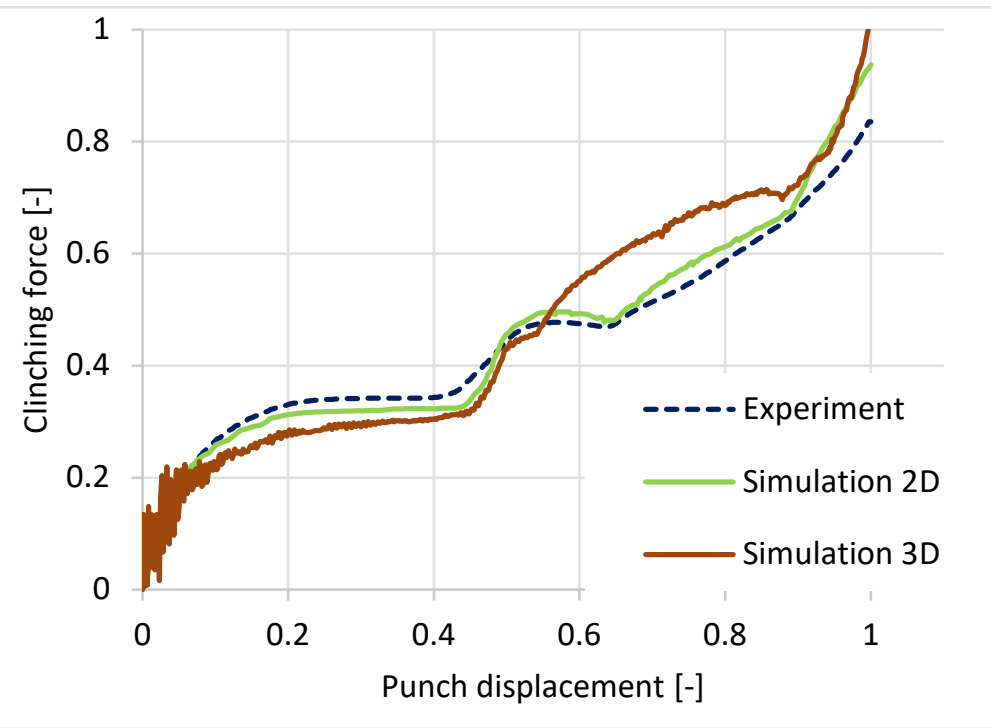

Fig. 7. Force displacement curves of the clinching process in experiment and simulation.
Secondly, the forcedisplacement curve is used to validate the simulation model. Picture 7 shows the experimentally measured forcedisplacement-curve of the clinching process and additionally two simulated force-displacement curves. One of the 2D model and one of the 3D model. The comparison of the 3 curves shows that both simulation models are able to simulate the forcedisplacement behaviour of the clinching process. The $2 \mathrm{D}$ model provides a slightly better match with the experimental curve than the 3D model.

Difference between 2D and 3D Models. In this paper two different methods were used to simulate the clinching process. First the implicit 2D model in the simulation software ANSYS and second the explicit 3D model in the simulation software LS-Dyna. This allows a comparison between both simulation techniques. In the 2D model, 1968 nodes and 1798 elements (at the beginning, the numbers change during the simulation because of the remeshing tool) are used to build up the forming process. The simulation time is round about $30 \mathrm{~min}$. In the 3D model there are 1692319 nodes and 1609790 elements (edge length in forming zone was $0.08 \mathrm{~mm}$ for both models) used. One forming process simulation takes $5078 \mathrm{~min}$. This shows how much more expensive a 3D simulation is in time and memory. Therefore, such an expensive simulation method should only be used when it is unavoidable like for the simulation of an offset between punch and preformed hole, when there is no longer an axial symmetry. 


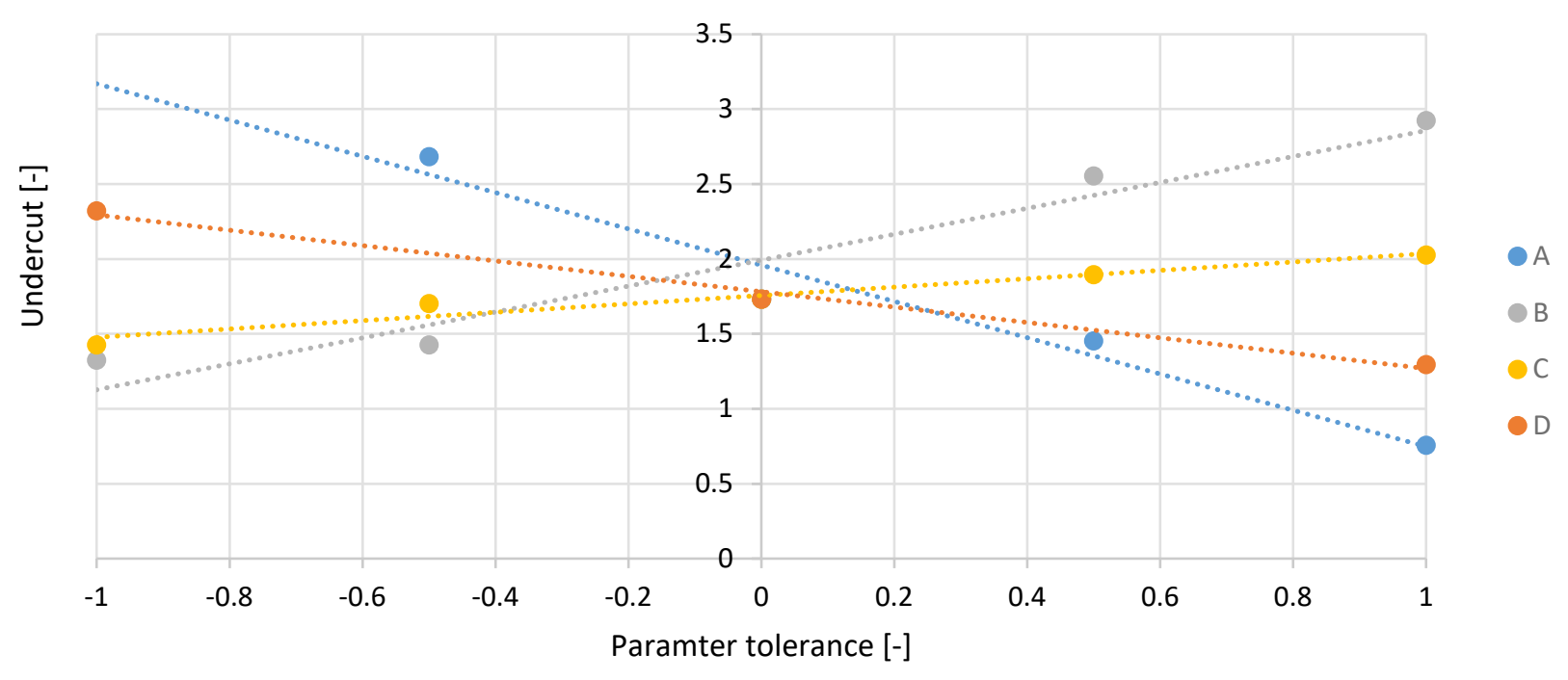

Fig. 8. Simulated influence of geometry tolerances of the preformed hole on the undercut of a clinched connection.

Process parameter depended interlock variation. In this work, the influence of three different process parameters, the geometry of the hole (described by the four Parameters A-D), the used material and an offset between the axes of punch and hole on the clinch quality was analysed.

Figure 8 shows the influence of the geometry parameters of the preformed hole on the undercut of the clinched connection. The values are normalised. Zero on the x-axis stands for the nominal value of the analysed geometry parameter. -1 and 1 stands for the maximum tolerancey that are allowed in negative and positive direction. The y-axis is normalized as well. The value of the interlock has to be higher than 1 so that the clinched connection is strong enough to ensure load transfer. The picture shows that there is a high sensitivity to parameter A, the diameter of the preformed hole. The largest allowed value for the diameter of the preformed hole leads to an insufficient undercut. Additionally there is no point marked at -1 . To simulate the forming process with the smallest allowed value for
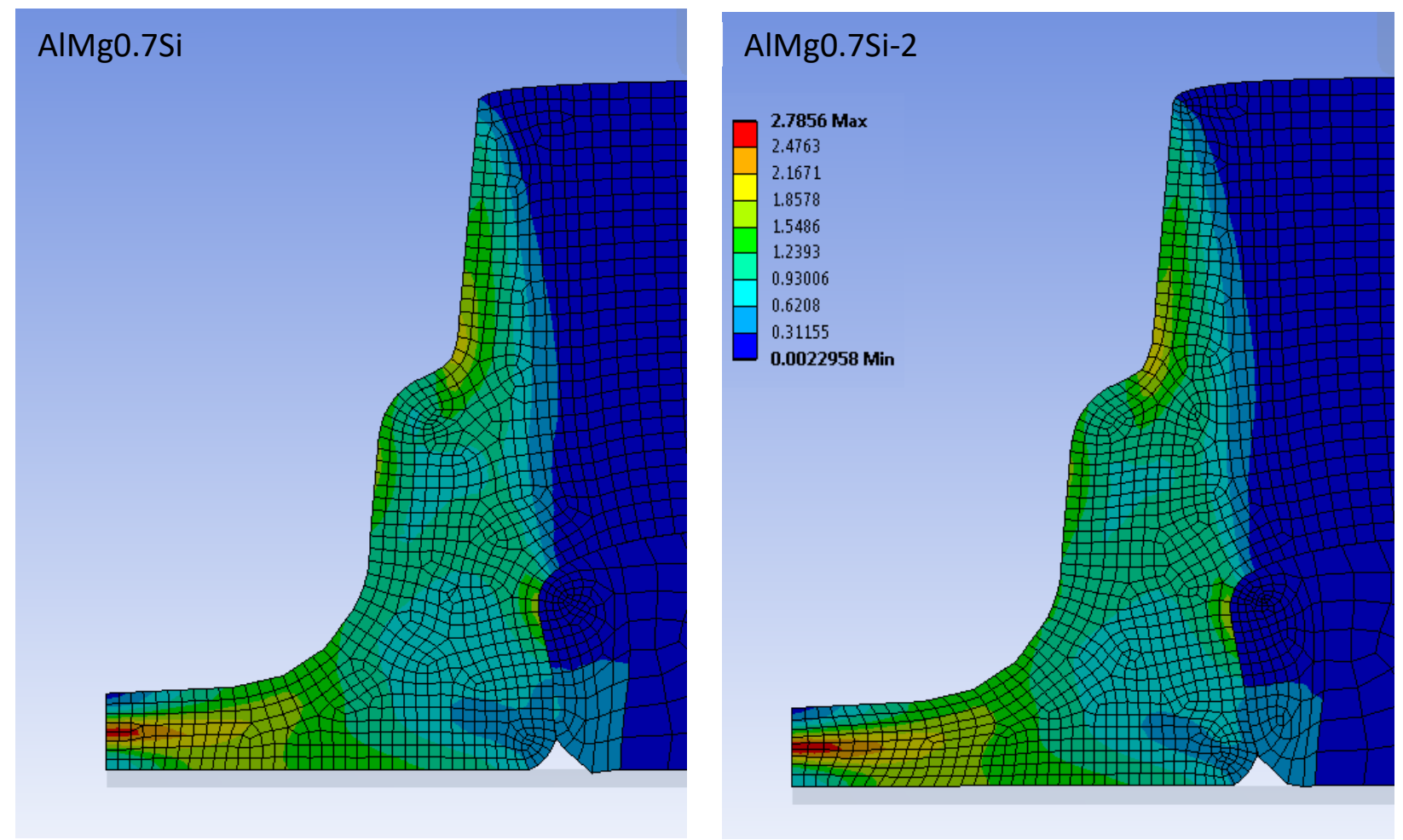

Fig. 9. Simulated undercut for two different material properties of the upper metal sheet. 
the diameter of the preformed hole was not possible because there was not enough space in the preformed hole of the bottom metal sheet for the material of the upper metal sheet to flow downward. Because of this, the simulation do not converge. So it was not possible to calculate an undercut for the smallest diameter. These two findings lead to smaller tolerances for the diameter of the preformed hole. For the other three parameters, the calculated interlock was higher than 1 for each possible value of the parameter. This means that these tolerances are ok. The gradient of the different process parameters in picture 8 shows which geometry parameter has the highest and the lowest influence on undercut width. Parameters $\mathrm{A}$ and $\mathrm{B}$ have a higher influence on the undercut than parameters $\mathrm{C}$ and D.

Picture 9 shows the simulated geometries for the two different analysed material properties shown in picture 3. The hardening curves show that there is a difference in deformability of the two materials. This fact can also be seen from the width of undercut after clinching. With the soft material an undercut of 2.5 was calculated compared to the undercut of 1.73 for the standard material.

The third process parameter influence, which was to be investigated in this work, was offset between the axes of punch hole and punch. With this process parameter the rotation symmetry is no longer valid. Therefore, the $2 \mathrm{D}$ model can no longer be used. Therefore, the examinations were carried out with the aid of the $3 \mathrm{D}$ model. As a result, the undercut can no longer be evaluated only at one point, but must be evaluated at several points. In this work, the undercut was evaluated in four different directions. As expected, the same values 1.7 for the undercut were obtained for the sample without offset in all four directions. This was not the case with the offset sample. Here, the values were 0 in offset direction, 1.5 on the opposite side and 1.7 for the two other orthogonal sides. So the average undercut was 1.2 for the specimen with the offset. The shown values were also normalized like the other given values for undercut in this paper. Figure 10 shows the difference between a clinch with and without offset. The picture shows a cross section of the clinched components. On the right
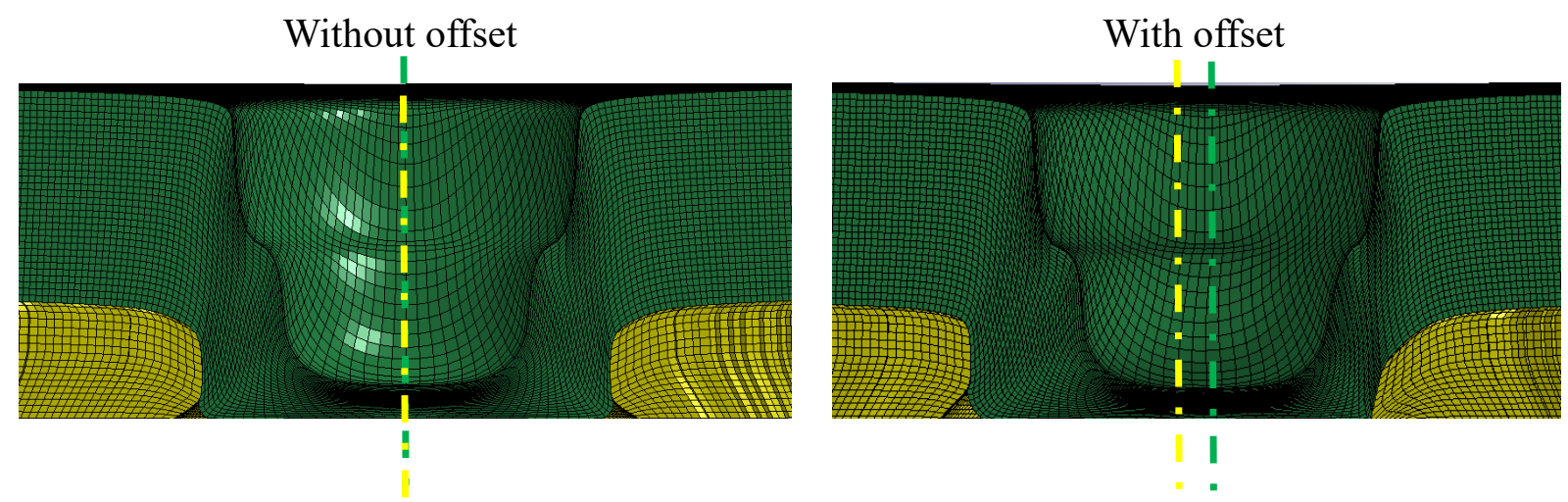

Fig. 10. Simulated clinch geometry without offset (left) and with Offset (right).

image, the punch has an offset in the right direction in comparison to the preformed hole. The inference from the picture is that due to this shift, there is no longer any formation of an undercut on the right side.

In this capture it was shown that there are a lot of process parameters which influence the quality of the clinched connection. Sometimes the value of undercut increases through changes in the process parameters but there are also a lot of process parameter changes which are responsible for a quality reduction and it has to be guaranteed that this reduction is not so low that the joint fails in the product.

\section{Conclusion}

In this work it could be shown that there is an influence of the process parameters on the quality of a clinched connection. For the investigation of the influence of individual process parameters on the joint connection, FE simulations were carried out. It has been shown that numerical simulation is a very effective tool to quantify process influences. With this method it was possible to study the different influences without performing many experiments. The work has shown that all four 
parameters examined have an influence on the clinch quality. It could be shown that there is a sufficiently large undercut for all given process parameter tolerances to carry the applicable loads. For the future, it is conceivable to combine the simulation in a simulative optimization program in order to optimize the quality of the entire clinch connection and to show possible interactions between individual process parameters.

\section{References}

[1] H. Fahrenwaldt, V. Schuler, J. Twrdek, Praxiswissen Schweißtechnik, Edition 5, Chapter 6 Fügen durch Umformen, (2014).

[2] E. Doege, B-A. Behrens, Handbuch Umformtechnik, Edition 1, Chapter 3.12 Fügen, (2007).

[3] TOX Pressotechnik, TOX-Joining-Systems, Product Catalogue, (2014).

[4] B. Awiszus, U. Beyer, M. Todtermuschke, F. Riedel, Flach-Clinchen - Simulationsbasierte Optimierung und Weiterentwicklung einer einseitig ebenen, einstufig gefügten Clinch-Verbindung, UTFscience, 2 (2009).

[5] B. Behrens, A. Bouguecha, M. Vucetic, S. Hübner, D. Yilkiran, Y. Jin, I. Peshekhodov, FEAbased optimisation of a clinching process with an open multiple-part die aimed at damage minimization in CR240BH-AlSi10MnMg joints, MATEC Web of Conferences 21, EDP Sciences (2015).

[6] M. Israel, Bewertung von Parameterstreuung beim Umformfügen, NAFEMS Magazin 2/2013, Ausgabe 26.

[7] P. Khrebtov, Neuartiges Verfahren zur Online-Prozessüberwachung beim Durchsetzfügeverbinden von Blechen, Dissertation, TU Clausthal (2011). 